\title{
Unobtrusive Contactless Cardiac Monitoring for Telemedicine and Ambient Assisted Living
}

\author{
Andres L Bleda ${ }^{1}$, Rafael Maestre ${ }^{1}$, Arcadio García ${ }^{2}$ \\ ${ }^{1}$ Technologic Centre of Furniture and Wood of Región de Murcia, Yecla (Región de Murcia), Spain \\ ${ }^{2}$ Hospital Virgen de la Arrixaca, Murcia, Spain
}

\begin{abstract}
During the last decades there has been a rapidly growing elderly population and the number of patients with chronic heart-related diseases has exploded. Many of them (e.g. congestive heart failure, some types of arrhythmia...) require a close medical supervision imposing a big burden to healthcare costs in most western economies. Telemedicine is clearly emerging as the real viable solution for health care to become sustainable by enabling medical monitoring at home without direct professional help, which would greatly reduce assistance costs. Specifically, continuous or frequent ECG monitoring is an important tool in the follow-up of many of these patients.

A cardiac monitoring device that requires no set-up or electrodes is highly desirable and of great value to the patients. Moreover, if that monitoring is always on and happens without the user even noticing it.

One such solution was researched in this work. Electric-field sensors have been integrated into the backrest of an armchair. This paper contains the details of the experimental study and final test results. A comparative discussion against traditional ECGs, as well as the merits of the new approach are also presented.
\end{abstract}

\section{Introduction}

Due to increasing life expectancy and decrease of fertility rates, the proportion of people aged 60 years and older is increasing faster than any other age group in almost all countries. According to WHO (World Health Organization) [1], the world's population of people 60 years old and over is forecast to reach 2 billion by 2050 . The population ageing can be considered a success of public health policies and socioeconomic development [2], but also a challenge to society, which must provide health services and try to improve old people functional capabilities, security and social participation.

The elderly is the most prone population group to suffer cardiovascular diseases (CVDs) such as heart attacks, strokes and arrhythmias. 17.7 million people die
TABLE I

EXPERIMENTAL SENSOR LOCATIONS AND CONFIGURATIONS

\begin{tabular}{cccccc}
\hline \hline \multirow{2}{*}{ Test } & Location & Filter & Textile & DRL & ECG Quality \\
\hline 1 & $1 R-1 L$ & $*$ LP & No & No & High \\
2 & $2 R-2 L$ & $*$ HP & No & No & Medium-low \\
3 & $2 R-2 L$ & $*$ LP & No & No & High \\
4 & $2 R-2 L$ & $*$ LP & Cotton & Yes & Very low \\
5 & $3 R-3 L$ & $*$ HP & No & No & Medium-low \\
6 & $3 R-3 L$ & $*$ LP & No & No & High \\
7 & $3 R-3 L$ & $*$ LP & Tergal & Yes & Very low \\
8 & $3 R-3 L$ & $*$ LP & Cotton & Yes & Very low \\
9 & $3 R-3 L$ & $*$ HP & Tergal & Yes & Very low \\
10 & $4 R-4 L$ & $*$ LP & Cotton & Yes & Medium \\
11 & $5 R-5 L$ & $*$ LP & Cotton & Yes & Medium-high \\
12 & $6 R-6 L$ & $*$ LP & Cotton & Yes & Very low
\end{tabular}

*LP: 6th order Low Pass filter with $40 \mathrm{~Hz}$ corner frequency

*HP: 3th order High Pass filter with $200 \mathrm{mHz}$ corner frequency

each year from CVDs (31\% of deaths worldwide) and $80 \%$ of these deaths are due to heart attacks and strokes [3].

Cardiac monitoring at home over longer periods of time can help to identify CVDs and prevent future cardiovascular problems, even risk of death can be predicted with the electrocardiogram (ECG) [4].

ECG measurements can be obtained with fixed electrodes on body or contactless systems [6-7]. On the one hand, fixed electrodes on body obtains reliable ECG measurements with low noise levels, but this is not a practical solution for long-term, everyday measurements. On the other hand, contactless systems are adequate for long-term monitoring, they are unobtrusive and can be fixed in the environment [8].

This paper includes an ECG monitoring furniture concept (that can be used by individuals during longer periods of time at their own homes), and the experimental results of contactless cardiac monitoring sensor tests developed for validating the sensor and the ECG acquisition.

\section{ECG furniture system design concept}

The elderly would like to maintain their independence, 
and live at their own homes for as long as possible. This population group spends most of their time at home and the use of resting furniture pieces such as armchairs, sofas or chairs is very common during the day, and so, they are an ideal object for integrating sensor systems in an unobtrusive and seamless way (the user does not perceive the existence of any non-familiar device in their usual environment). The design and integration of a ECG sensors system without direct skin contact in an armchair allows to obtain long-term, everyday measurements and continuous monitoring of user's cardiovascular state in a very convenient way. However, it should be noted that clothing acts as a physical barrier to these measurements and its impact should be carefully evaluated.

Figure 1 shows the cardiac monitoring furniture concept for ECG measurements (with no skin contact: a garment is used by its user), whose data is sent to an eHealth platform (where medical supervision is available).

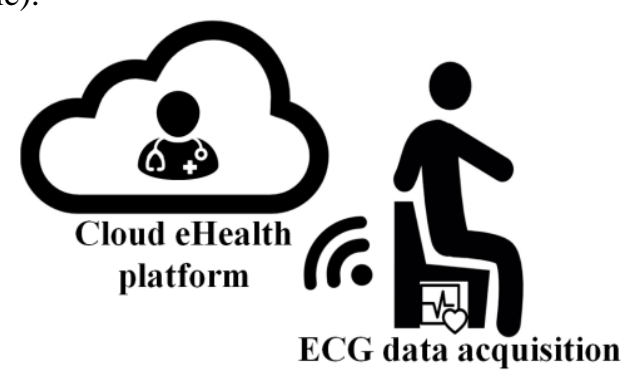

Fig. 1. ECG furniture system design concept

\section{Experimental setup}

The functionality of the designed system has been tested on an armchair that integrates the EPIC sensors for contactless ECG monitoring. The ECG signal has been acquired by a Plessey PS25003 EPIC demonstration kit [9].

\subsection{Hardware description}

A Plessey PS25003 EPIC demonstration kit was carry out the acquisition of the ECG. This kit is made up of:

- A main control box that includes a USB interface to a PC, a data acquisition system and filtering circuits (low-pass and $50 \mathrm{~Hz}$ rejection).

- Two PS25101 Plessey EPIC sensors [10]

\subsection{Software description}

A software application is provided for system configuration, acquired data filtering and real-time data visualization. Its interface is very similar to an oscilloscope, and the acquired signal can be digitally filtered with many different filter configuration options [9].

\subsection{System configuration}

In all the experiments the following configuration has been used:

- Control box: low-pass and $50 \mathrm{~Hz}$ rejection filters (Notch filter) are active with a gain of 10x.

- Software application:

- differential mode

$\circ$ filter:

- 6th order low-pass filter with a cut-off frequency (fc) of $40 \mathrm{~Hz}$

- $\quad$ or 3rd order high-pass filter with $\mathrm{fc}=200 \mathrm{mHz}$

○ sample rate: $250 \mathrm{~Hz}$

\subsection{Tested sensor locations}

Imperceptibility is a key aspect of this system, and furniture provides a physical support for the system elements and a perfect seamless integration. To validate the ECG data acquisition in furniture, it was necessary to place the Plessey sensors in different zones of the backrest of the armchair upholstery, where the back of the user will directly rest. Figure 2 shows the body areas that had been identified to have the highest potential for successful measurements.

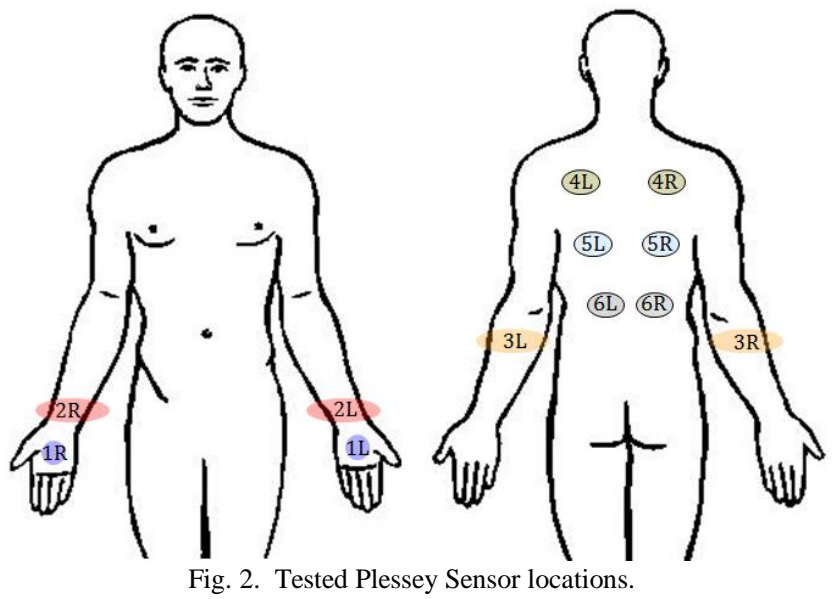

\section{Experimental results}

Table I contains a summary of the developed battery of tests. The location column indicates the Plessey sensor location according to Figure 2. The next columns state the filter type and type of textile contact (none, cotton or terylene. Textile garments were used in those body areas that are commonly covered by clothing (such as the back, the elbow or the wrist).

It was necessary to use a DRL on all body areas tested with the garment, otherwise the ECG signal was not 
detected. DRL stands for "driven right leg circuit" and is often added to biological signal amplifiers to reduce common-mode interference. In our case the DRL contact is implemented with a single piece of conductive textile placed under the right leg.

The following graphs show the obtained results of the tests included in table I:

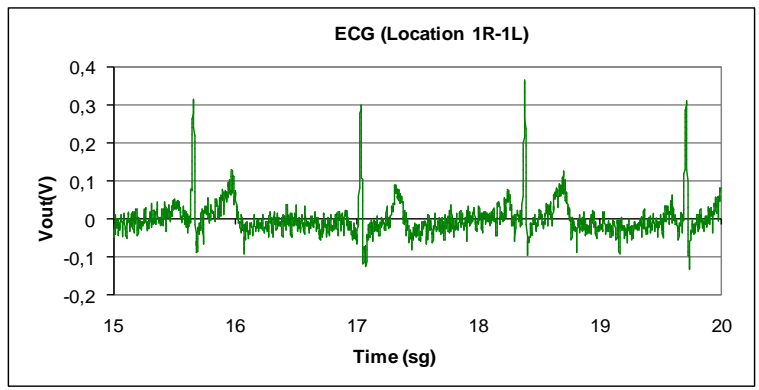

Fig. 3. Test 1 result.

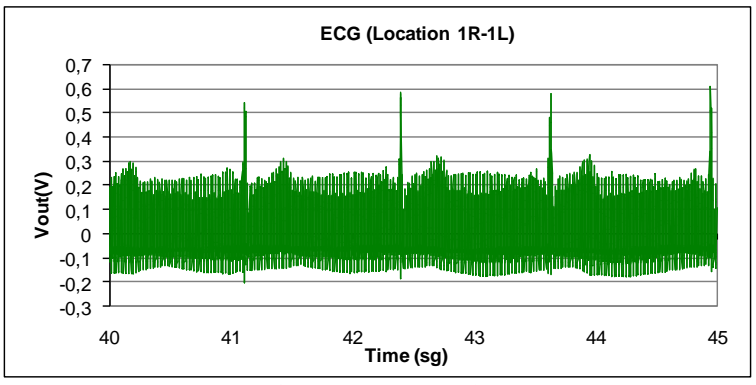

Fig. 4. Test 2 result.

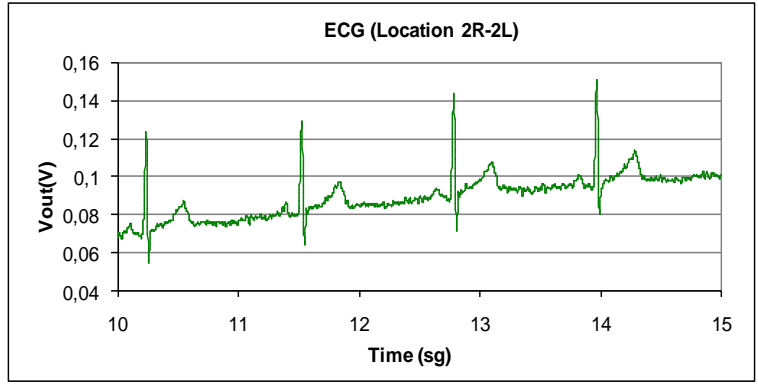

Fig. 5. Test 3 result.

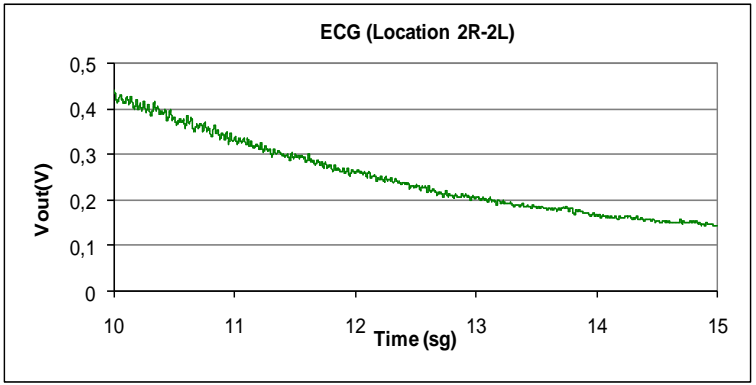

Fig. 6. Test 4 result.

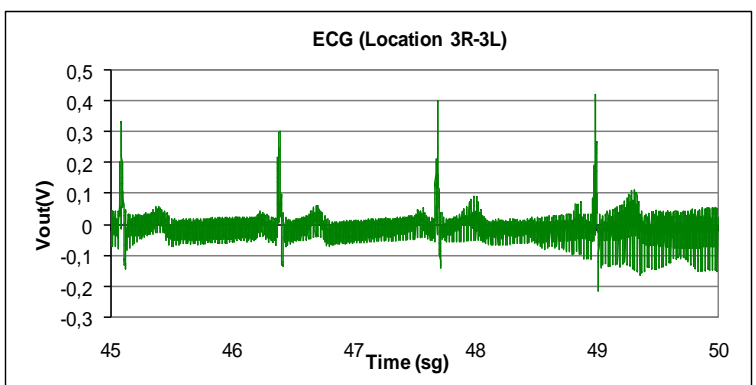

Fig. 7. Test 5 result.

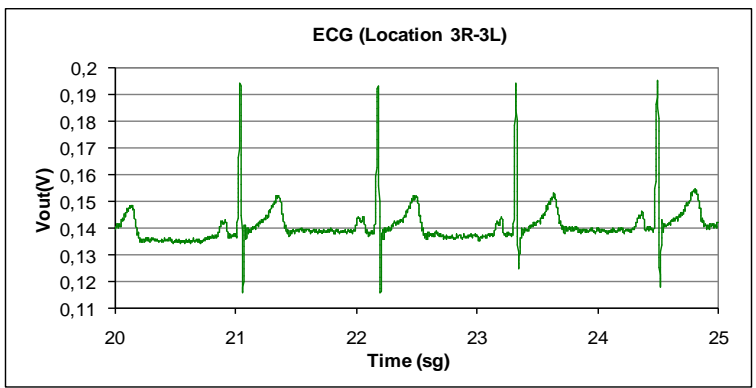

Fig. 8. Test 6 result.

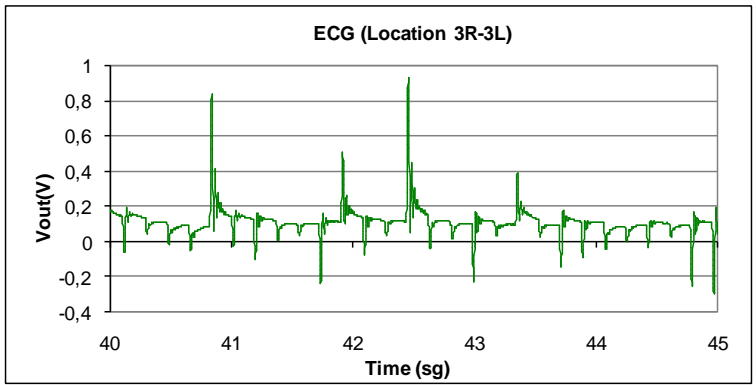

Fig. 9. Test 7 result.

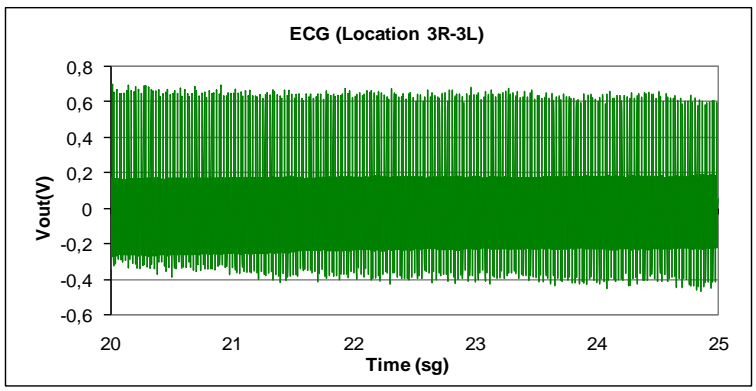

Fig. 10. Test 8 result.

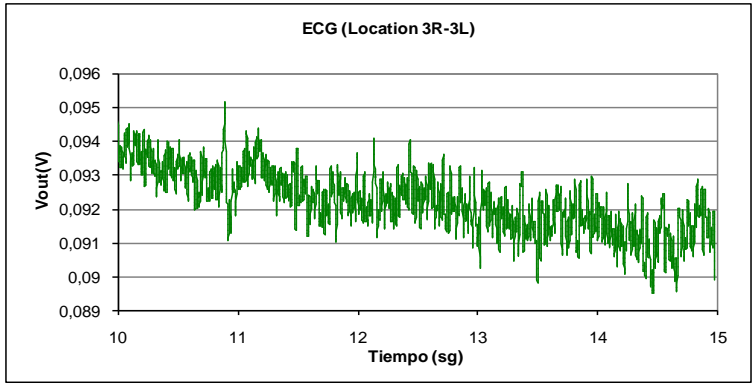

Fig. 11. Test 9 result. 


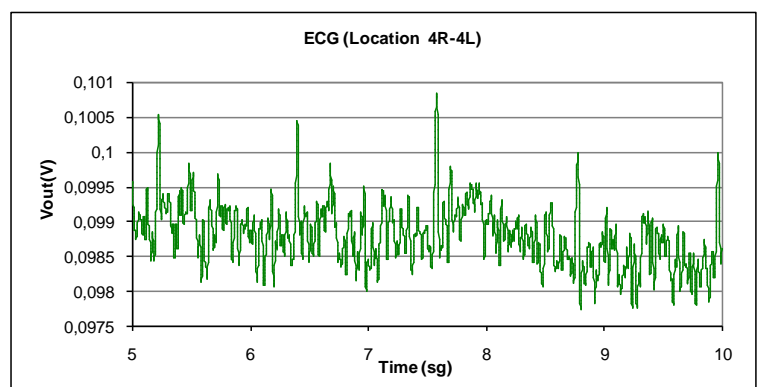

Fig. 12. Test 10 result.

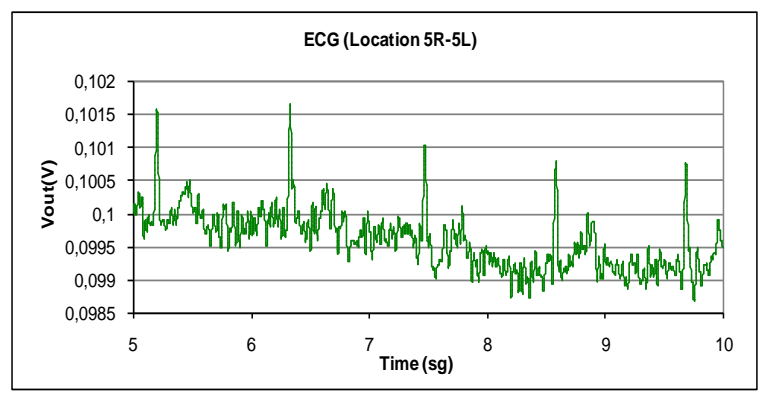

Fig. 13. Test 11 result.

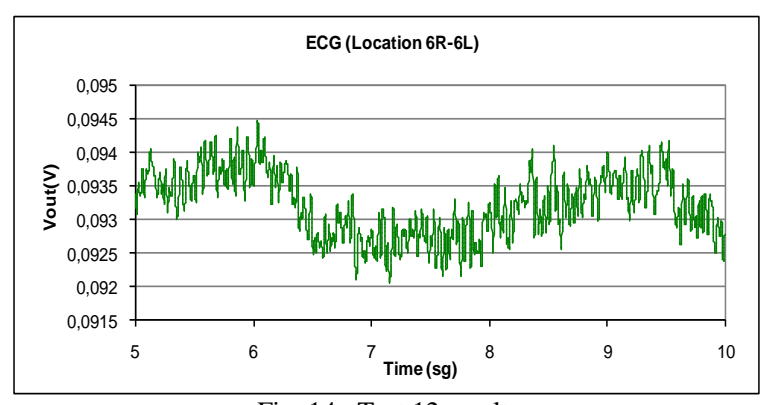

Fig. 14. Test 12 result.

\section{Conclusions}

As expected, tests with sensors in direct contact with the user's skin provide better quality than tests done without skin contact (i.e. through a garment). The areas of palm (1R-1L), wrist (2R-2L) and forearm (3R-3L) with direct contact of the user's skin provide a high quality.

Regarding experiments with contact through garment, data analysis confirms that cotton introduce less noise than any other textile type. According to the tests, when a garment is used, the ECG can be identified in wrist areas $(1 \mathrm{R}-1 \mathrm{~L})$, and in the upper and middle back areas $(4 \mathrm{R}-4 \mathrm{~L}$ and $5 \mathrm{R}-5 \mathrm{~L}$ ), but the other tested areas (forearms, $2 \mathrm{R}-2 \mathrm{~L}$, and low back, 6R-6L) introduce too much noise for extracting any useful ECG information.

\section{Acknowledgements}

We thank the staff of RGB medical devices and Virgen de la Arrixaca Hospital for their role in the collaborative development of the "Vitalmob" project, an R\&D project supported by the "Ministerio de Economia y Competitividad" from the Spanish Government under grant IPT-2012-1126-300000.

\section{References}

[1] World Health Organization (last access: 27/07/2018): http://www.who.int/news-room/fact-sheets/detail/ageingand-health

[2] World Health Organization. (2015). World report on ageing and health. World Health Organization.

[3] World Health Organization (last access: 27/08/2018): http://www.who.int/cardiovascular_diseases/en/

[4] Z Syed, et al. Computationally generated cardiac biomarkers for risk stratification after acute coronary syndrome. Science Translational Medicine, 3, 2011.

[5] Bleda, A. L., Maestre, R., Jara, A. J., \& Skarmeta, A. G. (2014). Ambient assisted living tools for a sustanaible aging society. In Resource Management in Mobile Computing Environments (pp. 193-220). Springer, Cham.

[6] Lim, Y. G., Kim, K. K., \& Park, S. (2006). ECG measurement on a chair without conductive contact. IEEE Transactions on Biomedical Engineering, 53(5), 956-959.

[7] Adib, F., Mao, H., Kabelac, Z., Katabi, D., \& Miller, R. C. (2015, April). Smart homes that monitor breathing and heart rate. In Proceedings of the 33rd annual ACM conference on human factors in computing systems (pp. 837-846). ACM.

[8] Fong, E. M., \& Chung, W. Y. (2013). Mobile cloudcomputing-based healthcare service by noncontact ECG monitoring. Sensors, 13(12), 16451-16473.

[9] Plessey PS25003 EPIC demonstration kit User guide (last access: 27/07/2018) http://www.plesseysemiconductors.com/wpcontent/uploads/ps25003-epic-development-kit-userguide.pdf

[10] Plessey PS25101 EPIC sensor (last access: 27/07/2018): http://www.plesseysemiconductors.com/wpcontent/uploads/ps25101-epic-sensor-development-sensorhigh-gain-datasheet.pdf

Address for correspondence.

Andrés L Bleda Tomás.

C/ Perales s/n

30510 Yecla

Murcia

Spain

al.bleda@cetem.es 